\title{
Design the Remote Monitor on the Scenic Spot Based on Integrated Sensor System
}

\author{
Xiaohui Wang ${ }^{1,2, a^{*}}$ and Lei Tian ${ }^{3, b}$ \\ ${ }^{1}$ Leisure Management College, Xi'an Eurasia University, Xi'an 710065, China \\ ${ }^{2}$ College of Tourism and Environment, Shaanxi Normal University, Xi'an 710119, China \\ ${ }^{3}$ School of Electronic Engineering, Xi'an University of Posts and Telecommunications, Xi'an 710121, \\ China \\ awxh2324@126.con, btianlei@xupt.edu.cn
}

Keywords: Sensor system; Embedded Linux; Remote monitoring; B/S mode.

\begin{abstract}
In order to monitor the scenic spot and transmit the collected data to the users, established the integrated sensing system based on the embedded Linux . Used the sensors to collect the different data and pictures and then they were transmitted by the wired and wireless mode. Set up the small Web server by the Boa (small Web server) and realized the integrated Web technology and CGI (Common Gateway Interface) program. According to the difference information of the scenic spot, the mobile platform collected the needed data and transmitted it to the control platform by the ZigBee wireless module and displayed in the embedded platform. The administrator can realize monitoring all the spots of the scenic and control the terminal equipments in the whole day.
\end{abstract}

\section{Introduction}

Today, all kinds of remote monitoring alarm system with its convenience, intuitive and variable attracted more and more people [1-3]. Especially with the rapid development of sensor technology, the remote monitoring system based on embedded device emerged, and has been widely used in all walks of life [4]. In this paper, a new B/S (browser and server) embedded remote monitoring system was developed and used in the scenic spot. Using the terminal equipment to monitor all aspect of the spot. At the same time, user can through the browser to control the terminal equipment. It reflected the human- computer interaction perfectly and greatly reduces the cost of the system development.

\section{System Structure and Function Design}

The Integrated Design of the System. The remote monitoring system was divided into three layers: the equipment layer, the transmission layer and the interface layer. The architecture of the system was showed in Fig. 1. In the terminal equipment layer, the ARM11 embedded board connected with USB camera [5], infrared sensor, sound sensor, light sensor and the temperature (humidity) sensor to monitor the spot information. Using the TCP/IP protocol [6], the information transmission layer sends the data which came from the terminal equipment layer through CGI interface to the user interface layer [7]. In the interface layer, the user login in and view the real time data from the transport layer through the browser. The user can input the control information from the interface and control the terminal sensor. Through the three layers' complete information, the system realized the purpose of the display and control the monitoring spots in real-time. 


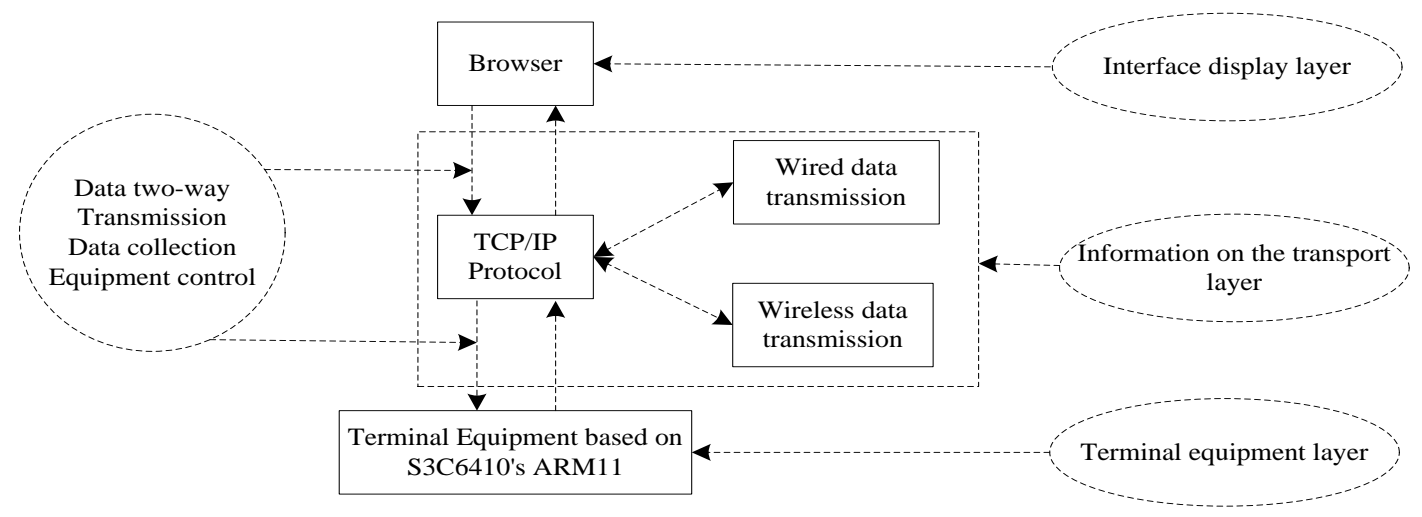

Figure 1. Structure diagram of the whole system

Hardware Structure of the Monitoring System. For the various considerations of safety, stable and transmission distance, the system transmitted the data in two ways; the wire transmission and the wireless transmission [8-9]. Different transmission modes corresponding to the different hardware and the hardware design as shown in Fig. 2.

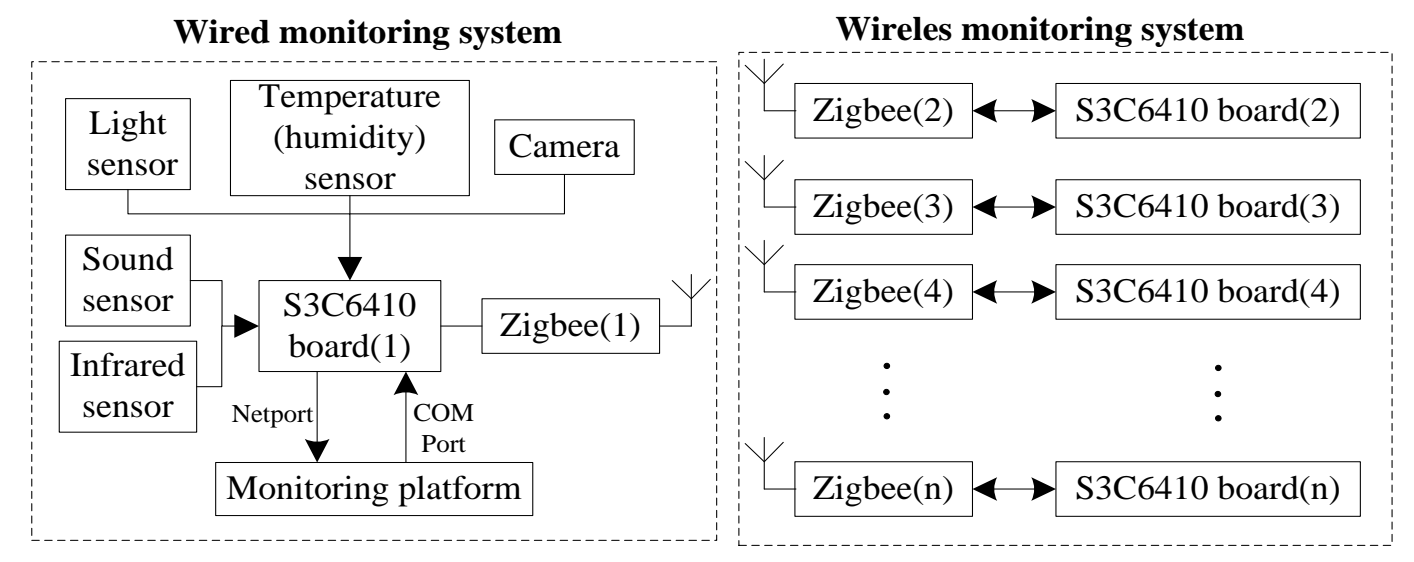

Figure 2. Hardware structure of the system

Hardware Design of the Wire Transmission. In the wired data transmission, using the No. 1S3C6410 embedded board as the core board, through the SDIO extended port connected with the human body infrared sensor, the sound sensor, the light sensor and the temperature (humidity) sensor. Using the USB camera acquire the spot information of monitoring site. The embedded board connected to the PC and builds the LAN (local area network) through the network interface. The embedded board was controlled and programmed through serial port COM1.

Hardware Design of the Wireless Transmission. During the period of the wireless transmission, the system can virtual the same S3C6410 development board as a mobile platform named No. 2, 3, $4 \ldots$ and etc. Because they have the same configurations with No. 1 embedded board, so the collected data can be send through the ZigBee module in real-time [10-11]. At the same time, the ZigBee module in No. 1 embedded board can be a receiver, the received data can be transmitted to monitoring platform.

\section{Software Structure of the Monitoring System}

Design of the Data Transmission Interface. In order to send the collected data to the user by a simple way and the user can view it directly. The most ideal method is using Web Server based on the Boa, through the CGI public interface, to implement the data exchange interface. In order to establish the Web server, the development environment was established on the PC platform through the Boa.

Design the Software Program. The software of this monitoring system was developed and operated based on the Linux platform. The system based on the B/S structure, using the TCP/IP 
protocol to transmit the data, to save the collected information through the established data sharing area and transmitted the sharing data to the monitoring platform. The specific process of the software system was showed in Fig. 3.

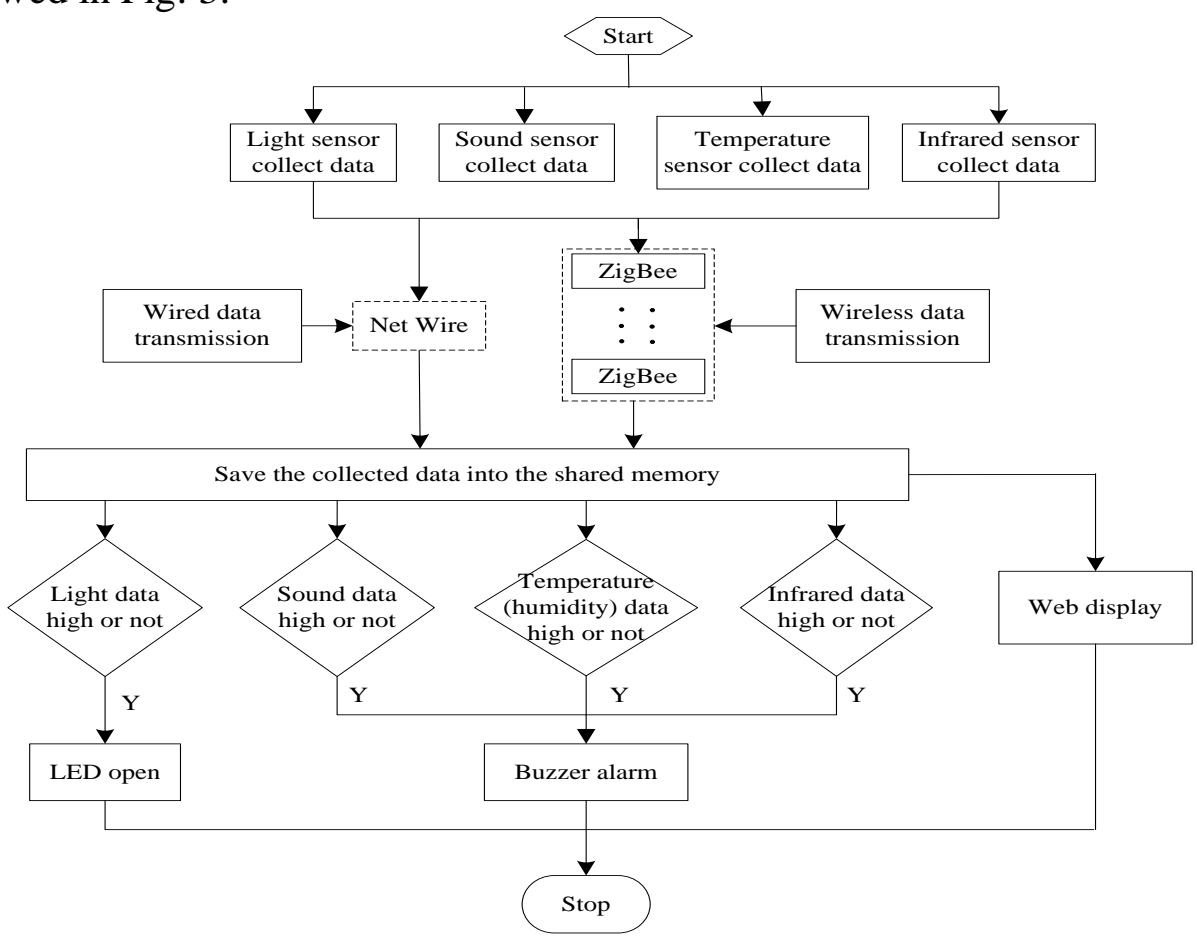

Figure 3. Structure of the software architecture

In order to obtain the data from the range of the monitored spot, the development board connected the USB camera, the temperature (humidity) sensor, the infrared sensor, the sound sensor and the light sensor with the SDIO port. Due to all the sensors need store the collected data constantly into the sharing area, the multi-thread coding technology based on Linux system would be needed.

Using sharing memory in the Linux system, created the shared database to store the collected data. With the configuration file, the information of the system login account and the upper and lower limits of the temperature (humidity) value of each sensor will be saved. Whenever refresh the web page, the data would be acquired from the sharing area. At the same time, read the input data configuration files from the web page and control the system.

\section{Simulation Results}

Terminal Setting. In this system, set up the web page interface, users can easily control the terminal equipment; terminal settings interface as shown in Fig. 4.

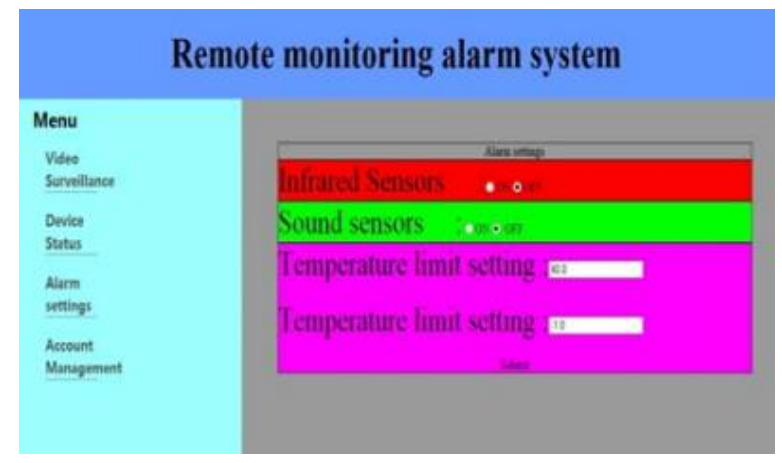

Figure 4. Terminal setting interface 
The user can regulate the switch of the infrared sensor and the sound sensor to control the sensors in the monitoring spots, and the upper and lower limits of the temperature can be manually set.

Terminal Display. In the test mode, the system got the environmental data through the variable sensors. Form the human body infrared sensor, the interface display "somebody" or "nobody". From the sound sensor, if the environment sound exceed the upper limits, the interface display "YES" or "NO". From the light sensor, if the obtained ambient light bigger than the setting value, the interface display "bright" or "weak". The true value of the temperature (humidity) sensors display in Fig. 5(a). The collected data was transmitted by the ZigBee wireless module and showed in Fig. 5(b).

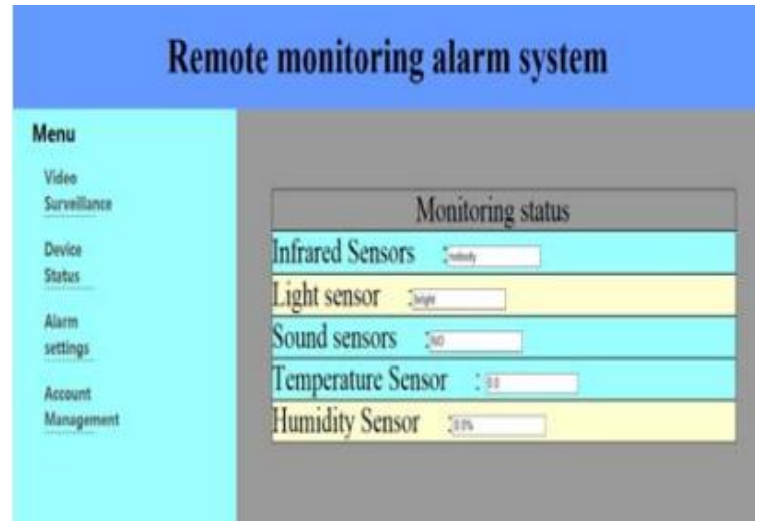

(a) True value of the sensor

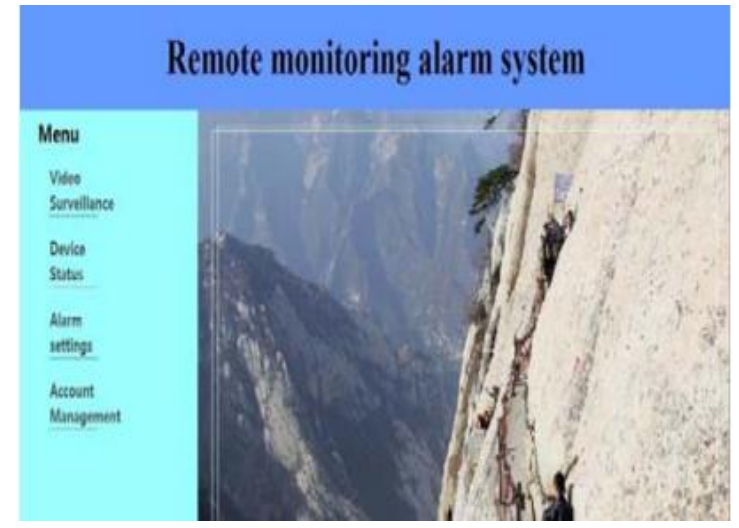

(b) The scenic spot monitor data

Figure 5. Display interface and feedback data

From the simulation results, the integrated sensor system can work very well in the monitoring spot. In this paper, the system was tested in the Huashan Mountain. The picture was showed in the touch screen in the embedded platform and told other users the scene of the situation. In the reality mountain spots, the wireless signals do not exist, so the mobile phone and the function of the mobile station will not be used. In this extreme situation, this integrated sensor system will help users to know the reality topography in time.

\section{Conclusion}

The remote monitoring system in this paper utilized the $\mathrm{B} / \mathrm{S}$ mode, reduced the development cost and the maintenance is convenient. The integrated sensor system collected all the data and transmitted to the main control platform. The multi-thread data transmission mode guaranteed the wired transmission system safety and stable. For the wider mobility, the wireless data transmission system was developed. In this system, take the advantages of both transmission modes, protect the normal operation of the monitoring system and extended the mobile area and saving the resources.

\section{Acknowledgement}

This work was partly supported by the project (2015EA03) supported by the key project of social science planning of Xi'an City, the Shaanxi Provincial Department of education scientific research project (No. 15JK1676) of China and the Youth Founded Project of Xi'an University of Posts and Telecommunications (101-0488).

\section{References}

[1] X.Ming. Sensors \& Transducers, Vol. 159(2013), No. 11,p. 431- 439.

[2] G.F.Qiao, G.M. Song, and Y. Wang, IEEE Transactions on Consumer Electronics, Vol. 59(2013), No.3, p. 562-570. 
[3] J. Kaczmarek, W. Miczulski and M.Kozioł. IEEE Transactions on Instrumentation and Measurement, Vol. 62(2013), No.10, p. 2828-2838.

[4] P.Aki, S. Ari and L. Risto. Energy and Buildings, Vol. 72(2014), No.4, p. 229-237.

[5] Y. Jian, D.S.Yin. Proceedings of the International Conference on Computer Science and Service System (Beijin, 2011), p. 1887-1890.

[6] M. Abrishamkar, M. Hussein and H. Maleki, Advanced Materials Research, Vol. 845(2014),p. 975-979.

[7] M.Eduardo, R.Manuel and Pérez Fernando.Sensors, Vol. 14(2013), No. 1, p. 416-430.

[8] C. Aldo, M. Fabrizio. Computers and Electronics in Agriculture, Vol. 101(2014), No.2, p. 118-127.

[9] D.W. He, W.X. Lin and N.Liu. IEEE Transactions on Smart Grid, Vol.4 (2013), No.4, p. 1870-1877.

[10]Z.Y.Jin, Z.X. Li and H.Jiang. Open Automation and Control Systems Journal, Vol. 6(2014), No. 1, p. 9-16.

[11]Z.C.Chen, C. Fabio. Structural Control and Health Monitoring, Vol. 21(2014), No.7, p. 1118-1136.

[12] A. D. Spacek, O. H. Ando Junior and J. Mota Neto. IEEE Latin America Transactions, Vol. 11(2013), No. 1, p. 512-517.

[13]H.H.Bian, Y.D.Wang. Sensors \&Transducers, Vol. 164(2014), No. 2, p. 114-119. 